\title{
A Solution to Control Suicides using Combined Overlap Block Fuzzy Cognitive Maps
}

\author{
P. Thiruppathi \\ Department of Mathematics \\ J.J. College of Engg.\& Tech., \\ Trichy-09, India.
}

\author{
N.Saivaraju \\ Department of Mathematics \\ Shri Angalamman College of \\ Engg. \& Tech. Trichy-05, India.
}

\author{
K.S. Ravichandran \\ Department of Information \\ Technology, SASTRA University, \\ Thanjavur -01, India.
}

\begin{abstract}
The imperative reason for suicide using Combined Overlap Block Fuzzy Cognitive Maps (COBFCMs), defined by W.B.VasanthaKandasamy, is analyzed in this paper. The Combined Overlap Block FCMs defined in this method becomes effective when the number of concepts can be grouped and are large in numbers. In this paper we analyzed the problem and find out the major reason for suicides using neutrosophic tool. This paper has five sections. First section gives the information about development of Fuzzy Cognitive Maps. Second section gives preliminaries of Fuzzy Cognitive Maps and Combined Overlap Block Fuzzy Cognitive Maps. In section three, we explain method of determining the hidden pattern. In the fourth section, we give the concepts of problem. Final section gives the conclusion based on our study.
\end{abstract}

Key words: FCMs, CBFCMs, Suicide

\section{INTRODUCTION}

Political scientist R. Axelrod [1] introduced cognitive maps for representing social scientific knowledge and describing the methods that are used for decision making in social and political systems. Then B. Kosko[2,3,4] enhanced the power of cognitive maps considering fuzzy values for the concepts of the cognitive map and fuzzy degrees of interrelationships between concepts. FCMs can successfully represent knowledge and human experience, introduce concepts to represent the essential elements and the cause and effect relationships among the concepts to model the behavior of any system. It is a very convenient, simple, and powerful tool, which is used in numerous fields such as socialeconomic and medical etc. Suicide, a social problem, is one of the leading causes of death in the world. The purpose of study is to identify the risk groups. Suicide is defined as deliberate killing of oneself. Suicides are most likely to occur during the periods of socio economic, family and individual crisis. Suicide prevention is an umbrella term for the collection of efforts of local citizen organizations, mental health practitioners and related professionals to reduce the incidence of suicide through the preventive and proactive measures. Moreover the data is an unsupervised one and also there is uncertainty in the concepts. Hence Fuzzy tool alone has the capacity to analyze these concepts. Hence it is chosen here.

\section{PRELIMINARIES}

Fuzzy Cognitive Maps (FCMs) are more applicable when the data in the first place is an unsupervised one. The FCMs work on the opinion of experts. FCMs model the world as a collection of classes and causal relations between classes.

Definition 2.1: An FCM is a directed graph with concepts like policies, events etc. as nodes and causalities as edges. It represents causal relationship between concepts.

Definition 2.2: When the nodes of the FCM are fuzzy sets then they are called as fuzzy nodes.

Definition 2.3: FCMs with edge weights or causalities from the set $\{-1,0,1\}$ are called simple FCMs.

Definition 2.4: The edges $e_{i j}$ take values in the fuzzy causal interval $[-1,1] . \mathrm{e}_{\mathrm{ij}}=0$ indicates no causality, $\mathrm{e}_{\mathrm{ij}}>0$ indicates causal increase $C_{j}$ increases as $C_{i}$ increases (or $C_{j}$ decreases as $C_{i}$ decreases). $e_{i j}<0$ indicates causal decrease or negative causality. $\mathrm{C}_{\mathrm{j}}$ decreases as $\mathrm{C}_{\mathrm{i}}$ increases (and or $\mathrm{C}_{\mathrm{j}}$ increases as $\mathrm{C}_{\mathrm{i}}$ decreases). Simple FCMs have edge values in $\{-$ $1,0,1\}$. Then if causality occurs, it occurs to a maximal positive or negative degree. Simple FCMs provide a quick first approximation to an expert stand or printed causal knowledge. If increase (or decrease) in one concept leads to increase (or decrease) in another, then we give the value 1 . If there exists no relation between two concepts, the value 0 is given. If increase (or decrease) in one concept decreases (or increases) another, then we give the value -1 . Thus FCMs are described in this way. Consider the nodes or concepts $\mathrm{C}_{1}, \ldots, \mathrm{C}_{\mathrm{n}}$ of the FCM. Suppose the directed graph is drawn using edge weight $\mathrm{e}_{\mathrm{ij}} \in\{0,1,-1\}$. The matrix $E$ be defined by $E=\left(e_{i j}\right)$, where $e_{i j}$ is the weight of the directed edge $\mathrm{C}_{\mathrm{i}} \mathrm{C}_{\mathrm{j}}$. $\mathrm{E}$ is called the adjacency matrix of the FCM, also known as the connection matrix of the FCM. It is important to note that all matrices associated with an FCM are always square matrices with diagonal entries as zero.

Definition 2.5: Let $C_{1}, C_{2}, \ldots, C_{n}$ be the nodes of an FCM. Let $A$ $=\left(a_{1}, a_{2}, \ldots, a_{n}\right)$, where $a_{1} \in\{0,1\} . A$ is called the instantaneous state vector and it denotes the on-off position of the node at an $\begin{aligned} \text { instant. } a_{i}=0 & \text { if } a_{i} \text { is off }=1 \\ a_{i}=1 & \text { if } a_{i} \text { is on, where } i=1,2, \ldots, n .\end{aligned}$ 
Definition 2.6: Let $\mathrm{C}_{1}, \mathrm{C}_{2}, \ldots, \mathrm{C}_{\mathrm{n}}$ be the nodes of an FCM. Let $\overrightarrow{C_{1} C_{2}}, \overrightarrow{C_{2} C_{3}}, \ldots, \overrightarrow{C_{i} C_{j}}$, be the edges of the FCM (i $\neq j$ ). Then, the edges form a directed cycle. An FCM is said to be cyclic if it possesses a directed cycle. An FCM is said to be acyclic if it does not possess any directed cycle.

Definition 2.7: An FCM with cycles is said to have a feedback.

Definition 2.8: When there is a feedback in an FCM, i.e., when the causal relations flow through a cycle in a revolutionary way, the FCM is called a dynamical system.

Definition 2.9: Let $\overrightarrow{C_{1} C_{2}}, \overrightarrow{C_{2} C_{3}}, \ldots, \overrightarrow{C_{i} C_{j}}$, be a cycle. When $\mathrm{C}_{\mathrm{i}}$ is switched on and if the causality flows through the edges of a cycle and if it again causes $\mathrm{C}_{\mathrm{i}}$, we say that the dynamical system goes round and round. This is true for any node $\mathrm{C}_{\mathrm{i}}$, for $\mathrm{i}=1,2, \ldots, \mathrm{n}$. The equilibrium state for this dynamical system is called the hidden pattern.

Definition 2.10: If the equilibrium state of a dynamical system is a unique state vector, then it is called a fixed point. Consider a FCM with $\mathrm{C}_{1}, \mathrm{C}_{2}, \ldots, \mathrm{C}_{\mathrm{n}}$ as nodes. For example let us start the dynamical system by switching on $\mathrm{C}_{1}$. Let us assume that the FCM settles down with $C_{1}$ and $C_{n}$ on, i.e. the state vector remains as $(1,0,0, \ldots, 0,1)$. This state vector $(1,0,0, \ldots, 0,1)$ is called the fixed point.

Definition 2.11: If the FCM settles down with a state vector repeating in the form $A_{1} \rightarrow A_{2} \rightarrow \ldots \ldots \rightarrow A_{i} \rightarrow A_{1}$, then this equilibrium is called limit cycle.

Definition 2.12: Finite number of FCMs can be combined together to produce the joint effect of all the FCMs. Let $\mathrm{E}_{1}, \mathrm{E}_{2}, \ldots$, $\mathrm{E}_{\mathrm{p}}$ be adjacency matrices of the FCMs with nodes $\mathrm{C}_{1}, \mathrm{C}_{2}, \ldots, \mathrm{C}_{\mathrm{n}}$, then the combined FCM $[5,6,7]$ is got by adding all the adjacency matrices $\mathrm{E}_{1}, \ldots, \mathrm{E}_{\mathrm{p}}$. We denote the combined FCM adjacency matrix by $\mathrm{E}=$ $\mathrm{E}_{1}+\mathrm{E}_{2}+\ldots+\mathrm{E}_{\mathrm{p}}$

Definition 2.13: Let $P$ be the problem under investigation. Let $\left\{\mathrm{C}_{1}, \mathrm{C}_{2}, \ldots, \mathrm{C}_{\mathrm{n}}\right\}$ be $\mathrm{n}$ concepts associated with $\mathrm{P}$ (n very large).Now divide the number of concepts $\left\{\mathrm{C}_{1}, \mathrm{C}_{2}, \ldots, \mathrm{C}_{\mathrm{n}}\right\}$ into classes $\mathrm{S}_{1}, \ldots, \mathrm{S}_{\mathrm{t}}$ where the classes are such that

(1) $\mathrm{S}_{\mathrm{i}} \cap \mathrm{S}_{\mathrm{i}+1} \neq \Phi$ where $(\mathrm{i}=1,2, \ldots, \mathrm{t}-\mathrm{t})$

(2) $\mathrm{US}_{\mathrm{i}}=\left\{\mathrm{C}_{1}, \cdots, \mathrm{C}_{\mathrm{n}}\right\}$
(3) $\left|S_{i}\right||\neq| S_{j} \mid$ if $i \neq j$ in general

Now we obtain the FCM associated with each of the classes $\mathrm{S}_{1}$, . .$S_{t}$. We determine the relational matrix associated with each $S_{i}$ Using these matrices we obtain a $n \times n$ matrix. This $n \times n$ matrix is the matrix associated with the Combined Overlap Block FCM(COBFCM) of blocks of same sizes.

Definition 2.14: Suppose $A=\left(a_{1}, \ldots, a_{n}\right)$ is a vector which is passed into a dynamical system $E$. Then $A E=\left(a_{1}^{\prime}, \ldots\right.$, $\left.a_{n}^{\prime}\right)$. After thresholding and updating the vector suppose we get $\left(b_{1}, \ldots, b_{n}\right)$. We denote that by $\left(a_{1}^{\prime}, a_{2}^{\prime}, \ldots, a_{n}^{\prime}\right) \rightarrow\left(b_{1}, b_{2}, \ldots\right.$, $b_{n}$ ). Thus the symbol $\rightarrow$ means that the resultant vector has been thresholded and updated. FCMs have several advantages as well as some disadvantages. The main advantage of this method it is simple. It functions on expert's opinion. When the data happens to be an unsupervised one the FCM comes handy. This is the only known fuzzy technique that gives the hidden pattern of the situation. As we have a very well known theory, which states that the strength of the data depends on the number of experts opinion we can use combined FCMs with several experts' opinions. At the same time the disadvantage of the combined FCM is when the weightages are 1 and -1 for the same $C_{i} C_{j}$, we have the sum adding to zero thus at all times the connection matrices $\mathrm{E}_{1}, \ldots, \mathrm{E}_{\mathrm{k}}$ may not be comfortable for addition. This problem will be easily overcome if the FCM entries are only 0 and 1.

\section{METHOD OF DETERMINING THE HIDDEN PATTERN}

Let $\mathrm{C}_{1}, \mathrm{C}_{2}, ., \mathrm{C}_{\mathrm{n}}$ be the nodes of an FCM, with feedback. Let $\mathrm{E}$ be the associated adjacency matrix. Let us find the hidden pattern when $C_{1}$ is switched on. When an input is given as the vector $A_{1}$ $=(1,0,0, ., 0)$, the data should pass through the relation matrix E. This is done by multiplying $A_{1}$ by the matrix E. Let $A_{1} E=$ $\left(a_{1}, a_{2}, ., a_{n}\right)$ with the threshold operation that is by replacing $a_{i}$ by 1 if $a_{i}>k$ and $a_{i}$ by 0 if $a_{i}<k$ ( $k$ is a suitable positive integer).We update the resulting concept, the concept $\mathrm{C}_{1}$ is included in the updated vector by making the first coordinate as 1 in the resulting vector. Suppose $\mathrm{A}_{1} \mathrm{E} \rightarrow \mathrm{A}_{2}$ then consider $\mathrm{A}_{2} \mathrm{E}$ and repeat the same procedure. This procedure is repeated till we get a limit cycle or a fixed point.

\section{CONCEPTS OF PROBLEM}

Using the linguistic questionnaire and the expert's opinion we have taken the following eight concepts $\left\{\mathrm{C}_{1}, \mathrm{C}_{2}, \ldots, \mathrm{C}_{8}\right\}$. The following concepts are taken as the main nodes for our problem. 
$\mathrm{C}_{1}$ - Poverty and unmanageable living cost (Economic Crisis).

$\mathrm{C}_{2}$ - More Working hours with poor salary.

$\mathrm{C}_{3}$ - Lack of commitment of breadwinners.

$\mathrm{C}_{4}$-Failure in business.

$\mathrm{C}_{5}$-Inability to deal with dept traps and torture from usurers.

$\mathrm{C}_{6}$-Government indifferences.

$\mathrm{C}_{7}$ - Stress due to mental, sexual and physical tortures in the working places.

$\mathrm{C}_{8}$-Lack of counselors to help persons out of depression.

Now we proceed on to apply the effect of combined overlap block FCM of equal length. Let us consider the eight concepts $\left\{C_{1}, C_{2}\right.$, $\left.\ldots, \mathrm{C}_{8}\right\}$. We divide these concepts into cyclic way of classes, each having just four concepts in the following way.

$\mathrm{S}_{1}=\left\{\mathrm{C}_{1}, \mathrm{C}_{2}, \mathrm{C}_{3}, \mathrm{C}_{4}\right\}, \mathrm{S}_{2}=\left\{\mathrm{C}_{3}, \mathrm{C}_{4}, \mathrm{C}_{5}, \mathrm{C}_{6}\right\}, \mathrm{S}_{3}=\left\{\mathrm{C}_{5}, \mathrm{C}_{6}, \mathrm{C}_{7}, \mathrm{C}_{8}\right\}$, $\mathrm{S}_{4}=\left\{\mathrm{C}_{7}, \mathrm{C}_{8}, \mathrm{C}_{1}, \mathrm{C}_{2}\right\}$.

The directed graph and the relation matrix for the class $S_{1}=\left\{C_{1}\right.$, $\left.\mathrm{C}_{2}, \mathrm{C}_{3}, \mathrm{C}_{4},\right\}$ given by the expert is as follows.

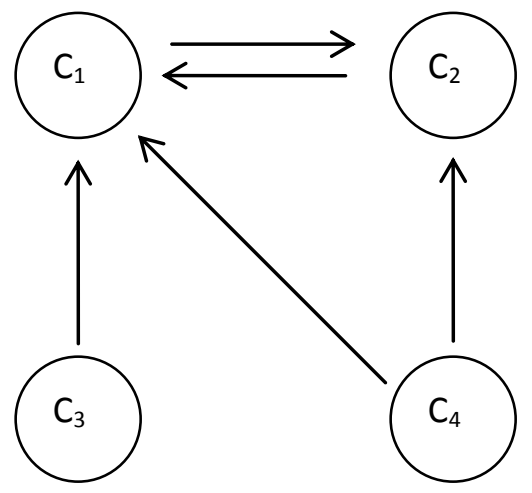

Figure 1

$$
\begin{aligned}
& \begin{array}{llll}
\mathrm{c}_{1} & \mathrm{c}_{2} & \mathrm{c}_{3} & \mathrm{c}_{4}
\end{array}
\end{aligned}
$$

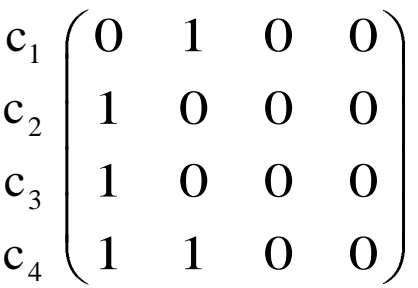

The directed graph and the relation matrix for the class $S_{2}=\left\{C_{3}\right.$, $\mathrm{C}_{4}, \mathrm{C}_{5}, \mathrm{C}_{6}$ \} given by the expert is as follows.

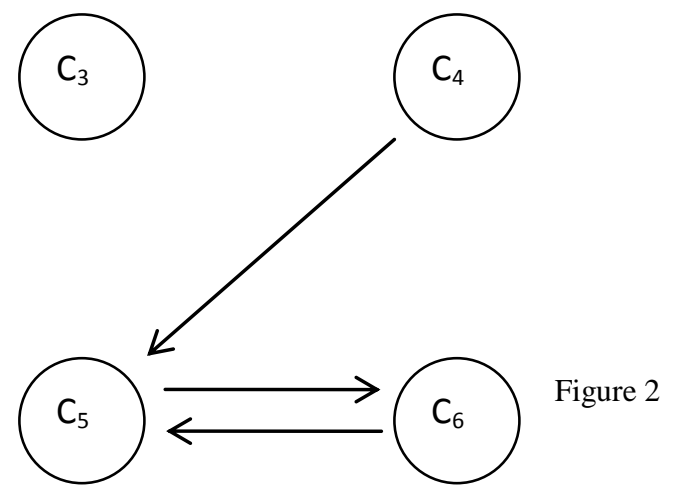

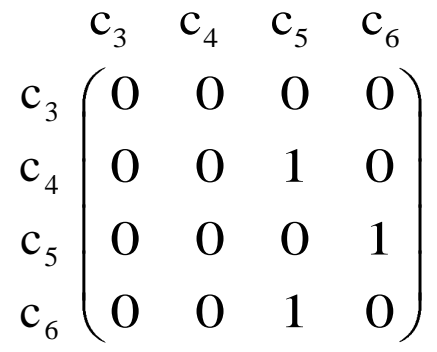

The directed graph and the relation matrix for the class $\mathrm{S}_{3}=$ $\left\{\mathrm{C}_{5}, \mathrm{C}_{6}, \mathrm{C}_{7}, \mathrm{C}_{8}\right\}$ given by the expert is as follows.

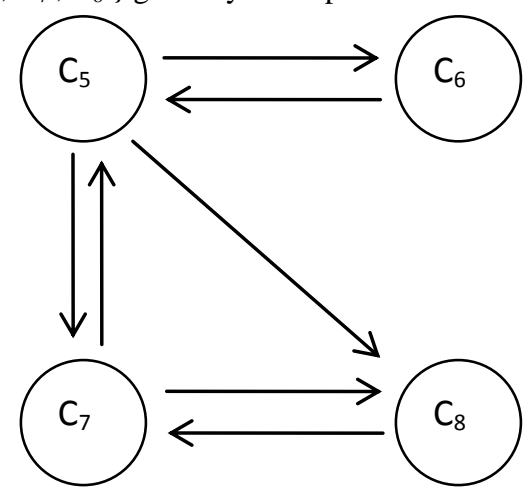

Figure 3

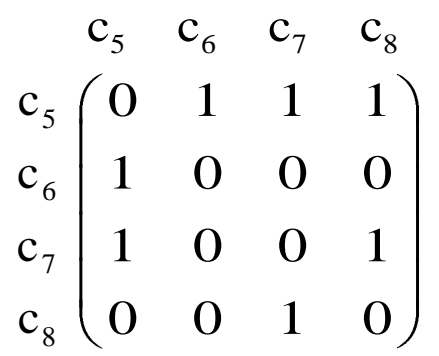

The directed graph and the relation matrix for the class $S_{4}=\{$ $\mathrm{C}_{7}, \mathrm{C}_{8}, \mathrm{C}_{1}, \mathrm{C}_{2}$ \} given by the expert is as follows.

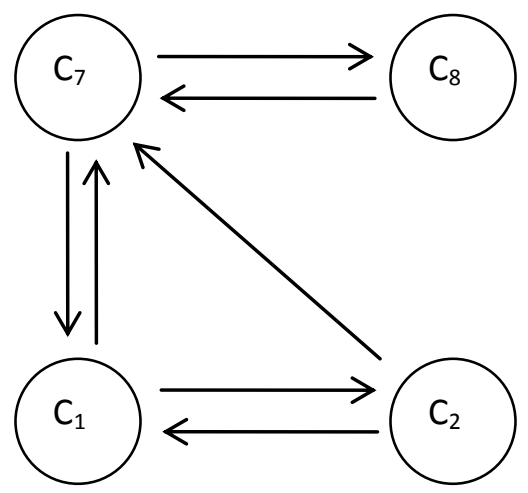

Figure 4 


$$
\begin{aligned}
& \begin{array}{llll}
\mathrm{c}_{7} & \mathrm{c}_{8} & \mathrm{c}_{1} & \mathrm{c}_{2}
\end{array} \\
& \begin{array}{l}
c_{7} \\
c_{8} \\
c_{1} \\
c_{2}
\end{array}\left(\begin{array}{llll}
0 & 1 & 1 & 0 \\
1 & 0 & 0 & 0 \\
1 & 0 & 0 & 1 \\
1 & 0 & 1 & 0
\end{array}\right)
\end{aligned}
$$

The combined directed graph and combined overlap block FCM of equal sizes is as follows:

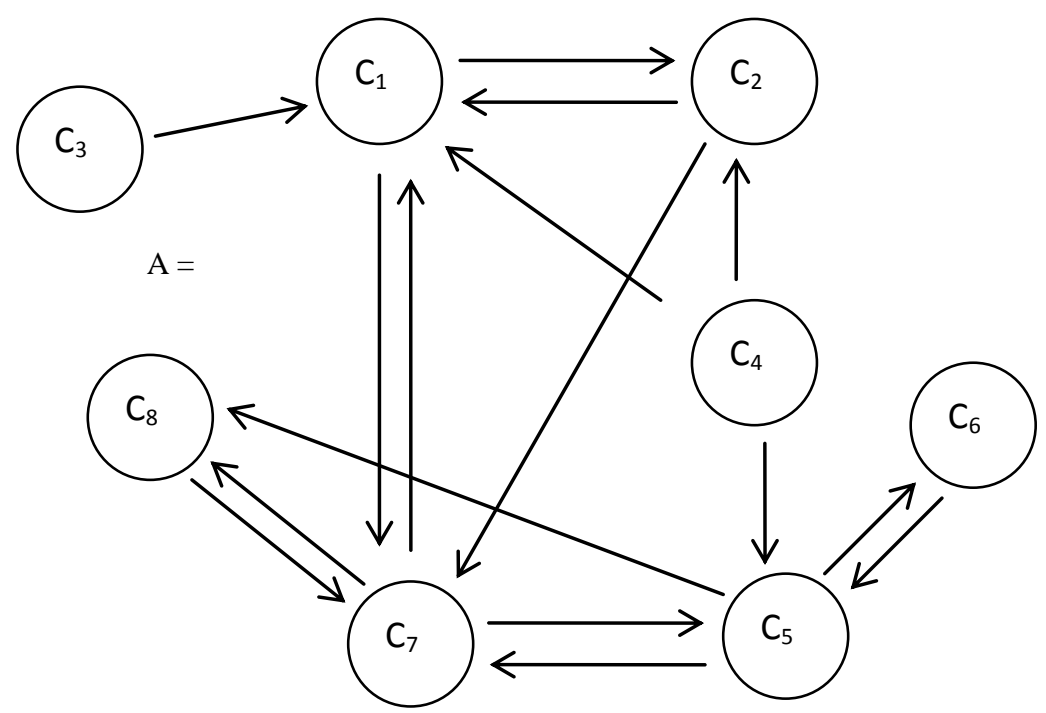

Figure.5

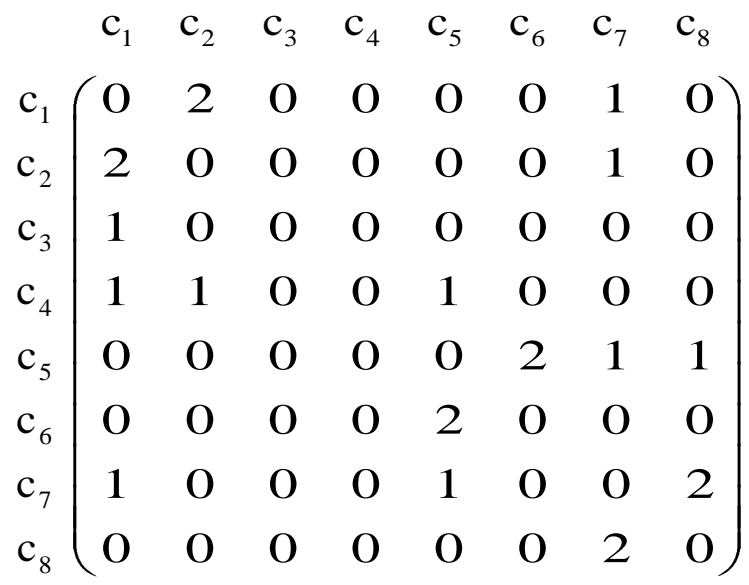

Now using the matrix A of the combined overlap block FCM, we determine the hidden pattern. Suppose the concept $\mathrm{C}_{8}$ is in the $\mathrm{ON}$ state and all other nodes are in the OFF state. Let the initial input

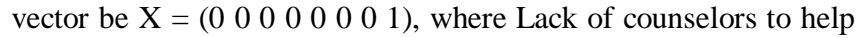
persons out of depression is taken as the ON state and all other nodes are in the OFF state.

The effect of $\mathrm{X}$ on the dynamical system $\mathrm{A}$ is given by:

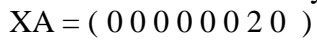

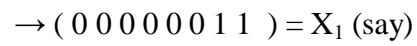

$$
\begin{aligned}
& X_{1} A=\left(\begin{array}{llllllll}
1 & 0 & 0 & 0 & 1 & 0 & 2 & 2
\end{array}\right)
\end{aligned}
$$

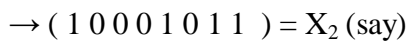

$$
\begin{aligned}
& X_{2} A=\left(\begin{array}{lllllll}
1 & 2 & 0 & 0 & 1 & 2 & 4
\end{array}\right)
\end{aligned}
$$

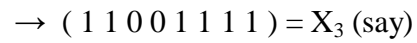

$$
\begin{aligned}
& X_{3} A=\left(\begin{array}{llllll}
3 & 2 & 0 & 0 & 3 & 25
\end{array}\right) \\
& \rightarrow(11001111)=X_{3}
\end{aligned}
$$

(Where $\rightarrow$ denotes the resultant vector after thresholding and updating)

$\mathrm{X}_{3}$ is the hidden pattern, which is the fixed point.

\section{CONCLUSION}

While analyzing FCM, when the concept $\mathrm{C}_{8}$, "Lack of counselors to help persons out of depression ", is in the $\mathrm{ON}$ state, the other concepts $\mathrm{C}_{1}, \mathrm{C}_{2}, \mathrm{C}_{5}, \mathrm{C}_{6}, \mathrm{C}_{7}$ are in the ON state. Also $\mathrm{C}_{3}$, and $\mathrm{C}_{4}$ are in the OFF state i.e When there is Lack of counselors to help Persons out of depression, there will be poverty and unmanageable living cost ( economic Crisis), working hours more with poor salary, inability to deal with dept traps and torture from usurers, government indifferences ,stress from mental, sexual and physical torture in the working places. Therefore "Lack of counselors to help persons out of depression" is the major reason for the suicides, since they can help the persons for a stress less life thereby avoid suicides.

\section{REFERENCES}

[1] Axelrod, R. (1976). Structure of decision: The cognitive maps of political elites. Princeton, NJ: Princeton University Press.

[2] Kosko, B., "Fuzzy Cognitive Maps", International journal of man-machine studies, January, (1986), 62-75

[3] Kosko, B. Hidden Patterns in Combined and Adaptive Knowledge Networks, Proc. of the First IEEE International Conference on Neural Networks (ICNN-86 (1988) 377-393.

[4] Kosko, B., Neural Networks and Fuzzy Systems: A Dynamical Systems Approach to Machine Intelligence, Prentice Hall of India, 1997.

[5] Vasantha Kandasamy and Smarandache Florentin, "Analysis of social aspects of migrant labourers living with HIV/AIDS using Fuzzy Theory and Neutrosophic Cognitive Maps", Xiquan, Phoenix. (2004)

[6] Vasantha Kandasamy W.B. and Victor Devadoss A., "Some New Fuzzy Techniques", Jour. of inst. of Math. \& Comp. Sci. (Math.ser.), Vol. 17, No.2, (2004 ).157- 160.

[7] Vasantha Kandasamy, W.B., and M. Ram Kishore. Symptom-Disease Model in Children using FCM, Ultra Sci., 11 (1999) 318-324. 